\title{
Helicopter Gearbox Bearing Fault Detection using Separation Techniques and Envelope Analysis
}

\author{
Linghao Zhou, Fang Duan, David Mba \\ School of Engineering \\ London South Bank University \\ London, U.K. \\ zhoul7@1sbu.ac.uk, duanf@1sbu.ac.uk, mbad@1sbu.ac.uk
}

\author{
Michael Corsar, Matthew Greaves, Suresh Sampath \\ CSAIC, and Centre for Propulsion \\ Cranfield University \\ Milton Keynes, U.K. \\ m.r.corsar@cranfield.ac.uk, m.j.greaves@cranfield.ac.uk, \\ s.sampath@cranfield.ac.uk
}

\author{
Faris Elasha \\ School of Mechanical, Automotive and Aerospace Engineering \\ Coventry University \\ Coventry, U.K. \\ ac1027@coventry.ac.uk
}

\begin{abstract}
The main gearbox (MGB) is a crucial part of a helicopter. MGB bearings suffer intensively from stress and friction during flights hence concerns for their health condition and detecting potential defects become critical for the sake of operation safety and system reliability. In this study, bearing defects were seeded in the second epicyclic stage bearing of a commercial Class A helicopter MGB. Vibration and tachometer signals were recorded simultaneously for the purpose of fault diagnosis. The tests were carried out at different power and speed conditions for various seeded bearing defects. This paper presents a comparison of signal processing techniques employed to identify the presence of the defects masked by strong background noise generated from an operation helicopter MGB.
\end{abstract}

Keywords- MGB; Bearing Fault Diagnosis; Signal Separation; Kurtogram; Envelope Analysis

\section{INTRODUCTION}

The main gearbox (MGB) is responsible for converting high input speed with low torque from engines to low speed with high torque in order to drive rotor and other accessory systems. It plays a vital important role in the dynamic drive train system which guarantees that appropriate torque can be provided to rotors to fulfil different flight tasks. The transmission nature of such system dictates that the friction heat and large mechanical force applied to MGB components are significant, weakening them in long term and eventually causing failure. Therefore, successful diagnosis of incipient defects of MGB components could not only enhance the system reliability and functionality, but also be life-saving. In this study, the fault diagnosis is focused on MGB bearings defects, which constitutes one of the most common failure modes.
Vibration-based feature extraction techniques have been widely adopted in detecting bearing faults [1]. These techniques utilise the rotating characteristics of targeted components, monitor their vibration levels, and observe for significant fault features either in time domain or frequency domain [2]. However, it must be pointed out that in most cases, simple observation of time-domain signals could rarely give any clear sign of faults as different frequency components tend to smear the raw signals and make all the information ambiguous. Fast Fourier Transform (FFT) based feature extraction techniques thus are developed to transfer signals from time domain to frequency domain, where different frequency components are relatively more distinguishable and fault frequency could be hence detected.

Although FFT based techniques are more efficient and accurate for fault diagnosis vastly compared with examining raw vibration signal, the detection process could still be cumbersome. This is especially true when it comes to MGB fault diagnosis. MGB is a highly sophisticated machine with large numbers of components meshing and rolling, making the output signal noisy and complex hence sometime FFT based techniques fail to detect faults related features [3, 4]. For this reason, technique of kurtogram combined with envelope analysis which has strong noise-cancelling capability's for exposing bearing fault features is utilised in this paper for detecting bearing fault. The data used were collected from a special designed test rig built with a commercial Class A helicopter gearbox.

\section{TEST RIG AND EXPERIMENT SETUP}

The test was carried out on a SA 330 Puma helicopter MGB with seeded defects located at bearing outer race. Gearbox layout and parameter ratios are illustrated in Fig. 1 [5]. 


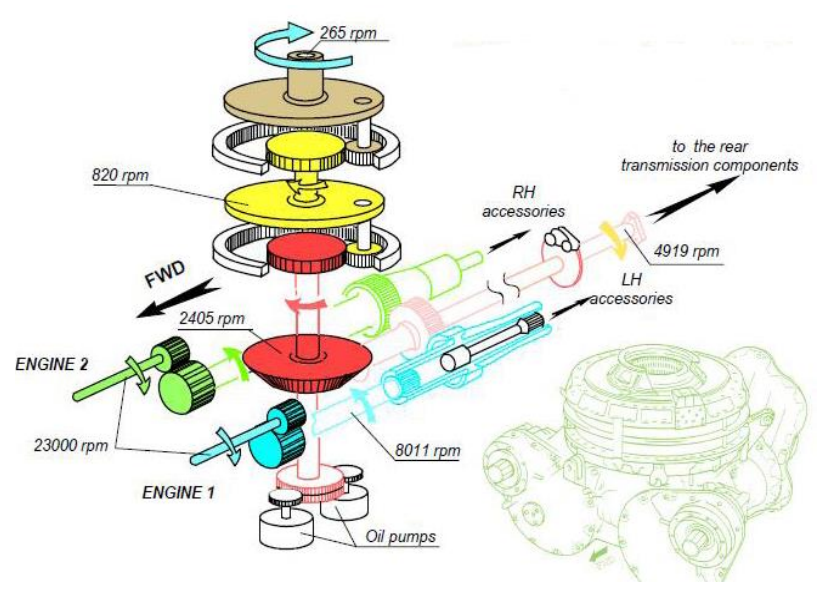

Figure 1. Gearbox internal layout from [5].

The experiment was well planned with different phases, including commissioning, seeded fault tests, dry bearing tests. In this paper, seeded fault tests are specifically focused, with no other specifications, all tests below are referred to seeded fault tests only. Tests under different output power and speed conditions were performed for MGB 2nd epicyclic stage bearing with 3 different conditions, namely healthy, minor damaged and major damaged outer race respectively. The maximum output of $1760 \mathrm{~kW}$ was designed to simulate the flight regime of $110 \%$ take-off power, while a minimum output of $92 \mathrm{~kW}$ represented the condition of helicopter being ground-idle. Maxi-mum speed output reached $275 \mathrm{rpm}$ at second epicyclic carrier of MGB, reflecting an engine input speed of $23000 \mathrm{rpm}$. Other tests conditions such as 'ground idle', which has lowest load and minimum speed output, and ' $120 \%$ max continuous power' condition were also undertaken for post processing. In case of signal saturation, vibration sensors with sensitivity of $10 \mathrm{mV} / \mathrm{g}$ were adopted (PCB $352 \mathrm{C} 03$ and Endevco 6251M4) and placed at six carefully chosen locations. Vibration signal was sampled at $51.2 \mathrm{kHz}$ before anti-aliasing filtered at $25.6 \mathrm{kHz}$. Bench parameters including output torques and a 60-pulse tachometer signal were recorded simultaneously for the purpose of post processing. Specific experiment setup and data collections schemes are fully described in [6].

\section{Signal Processing TeChNiques AND ANALysis}

As explained above, the ultimate goal of feature extraction is exposing potential fault frequencies and comparing them with theoretical fault frequencies for matches. Calculations of notable MGB bearings fault frequencies could be easily established based on bearing's geometry [7].

In this study, maximum power setting of $1760 \mathrm{~kW}$ is considered for processing and analysis. In all three conditions (healthy, minor damaged and major damaged), maximum output speed of $275 \mathrm{rpm}$ from $2^{\text {nd }}$ epicyclic stage is adopted for determining ball pass frequency of outer races (BPFO). According to equation (2), the calculated BPFO which will be used for identifying existence of bearing outer race fault is:
TABLE I. FAULT FREQUENCY AND MACHINE CONDITIONS

\begin{tabular}{|l|c|c|c|}
\hline Fault Condition & Load $(\mathbf{k W})$ & Speed (rpm) & BPFO (Hz) \\
\hline Major and Minor & 1760 & 275 & 101.6 \\
\hline
\end{tabular}

Notice that due to random slip of rolling element, the calculated BPFO can have small degree of deviation.

Examination of time-domain signal is employed first, and a speed fluctuation could be detected with the aid of tachometer signal (Fig. 2).

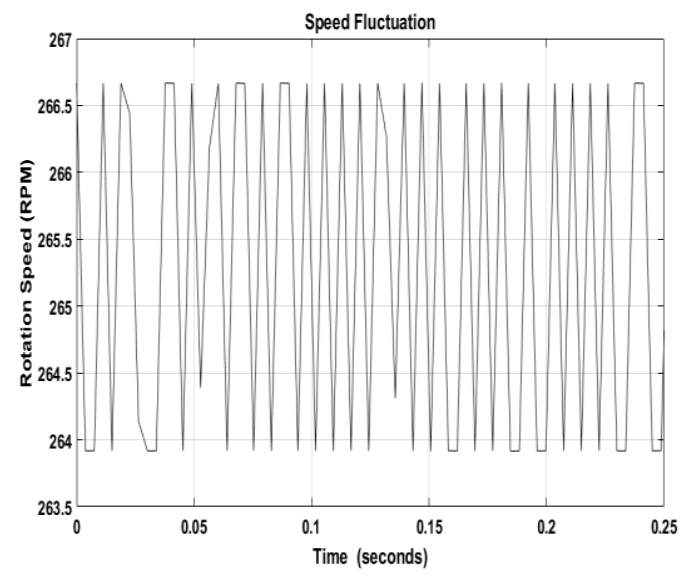

Figure 2. Detected speed flucuations.

It is evident that speed fluctuation could bring unwanted smearing of discrete frequency components [2]. Although from Fig. 2, the estimated speed fluctuation is approximately $1 \%$, and the disturbance might not be critical, a pre-processing is also applied for best accuracy. Order tracking has been adopted as a common tool $[2,8,9]$ for cancelling effects of speed fluctuation. The method is achieved normally by angular resampling and interpolation, in order to reach a uniform increment in shaft rotating angle. Entire procedure could include but not limited with digital oversampling, anti-aliasing, angular resampling, scaling, digital decimation and filtering. The processed results would ideally be data equally sampled not in time-domain, but in angular domain.

\section{A. Signal Separation}

As previously stated, vibration signals collected inside an operational MGB can be extremely noisy, what makes it even worse is strong gear meshes that mask the target bearing signals. A good counter is signal separation that aims at separating gear mesh signals and bearing signals, also improves signal-to-noise ratio. Many separation methods have been developed such as linear prediction, adaptive/self-adaptive noise cancellation (ANC/SANC), time synchronous averaging (TSA) and so on. For easier application purposes, discrete random separation (DRS) is chosen in this paper.

DRS developed by Antoni and Randall, acts very similar to SANC algorithm, gaining significant simplicity in implementation with a slight compromise of filter performance [4]. DRS utilises the difference of signal correlations between gear and bearing signals, as former are more periodic hence 
theoretically have longer correlation time. Bearing signals on other hand, due to the presence of roller slip, generally have certain randomness which makes bearing signal less 'predictable', hence have less correlation time. The main idea of DRS is to cut data $\mathrm{Y}(f)$ into segments, each of which is used for performing frequency response function $\mathrm{H}(f)$ with delayed signal $\mathrm{Y}_{\Delta}(f)$. These frequency response functions are eventually averaged to get a quotient of expectations ' $E$ ', which gives a series of values theoretically scattered from 0 to 1 , where 0 means signal components not correlated while 1 means signal components still correlated after delay. These values can then be transformed back to time domain to form a filter to apply on origin data directly therefore uncorrelated random signals could be filtered out. The definition equation of DRS is described below:

$$
H(f)=\frac{\mathbb{E}\left\{Y(f) Y_{\Delta}^{*}(f)\right\}}{\mathbb{E}|Y(f)|^{2}}
$$

Separation result is demonstrated in Fig 3, data used are from $1760 \mathrm{~kW}$ load, and $275 \mathrm{rpm}$ speed minor fault condition. It is quite obvious that the chaotic original signal has become more 'modulated' and impulsive after separation, SNR has improvements.

DRS separation is meant for gear/bearing signal separation, another algorithm that complements noises cancellation i.e. separate impulsive signals and stationery noise, has to be adopted. Kurtosis has been established as a gauge for vibration health condition monitoring of rotating machinery for years. It is defined as the fourth standardised moment of the signal, with capabilities of reflecting how flat or peaky the signal is. Spectral kurtosis (SK) is kurtosis of different frequency components of the signal. SK was first introduced by Dwyer [10] for separation of non-Gaussian components of a signal in its frequency domain. As short-time-frequency-transform (STFT) based, SK measures impulsiveness of the signal in different frequency band, thus the most impulsive frequency components which might contain fault signatures could be identified and extracted. SK has been extensively used in fault detection and condition monitoring [11-12]. A very thorough definition and application of SK has been demonstrated by Antoni in [13], where SK has been statistically defined in equation (2) as fourth-order normalised cumulant of the signal, where $\operatorname{Env}(n, f)$ represents complex envelope of signal at frequency $f$.

$$
S K=\frac{\left\{\left|\operatorname{Env}(n, f)^{4}\right|\right\}}{\left\{|\operatorname{Env}(n, f)|^{2}\right\}^{2}}-2
$$

From the description, it is indicated that the choice of frequency band or the frequency resolution is critical. In order to enhance efficiency in determining appropriate frequency resolution, Kurtogram is developed, which display SK as a function of both frequency and frequency resolution. In this way, an optimal band pass filter could be designed to extract signals with maximum impulsiveness in certain frequency bands and centre frequencies. Antoni has also suggested a fast kurtogram algorithm [14] to lessen the complicated computational work of kurtogram exploring the entire plane of frequency/ resolution.
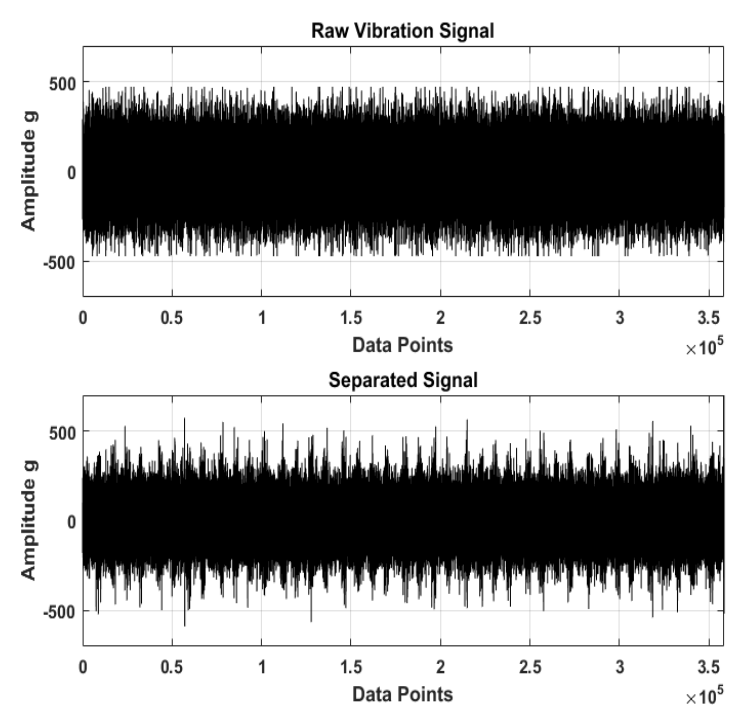

Figure 3. Separation of vibration data.

\section{B. Envelope Analysis}

Envelope analysis has been established as benchmark method for dealing with bearing fault diagnosis. The advantages of envelope analysis is dragging high frequency signal components that usually contains structural resonances to low frequency range, thus periodic signatures are more easily detected through examining envelope of the signal. The procedure of envelope analysis is described in [15, 16]. Generally, the process of performing envelope analysis comprises of following procedures: first of all, determining appropriate high frequency band to be filtered, which should contain most impulsive components. Band-passing the chosen frequency range, and implementing Hilbert transform so that the signal is converted to analytic signal. The absolute value of the transformation results is the envelope of Bandpass-filtered signal. At last, traditional inspections on frequency components of enveloped signal could be carried out to search for desired faulty frequency components.

One problem when applying envelope analysis is that selecting frequency bands for filtering might be challenging. Impacts due to defects could excite resonance at higher frequencies. With the aid of Kurtogram, it is possible to identify these structural resonance frequencies and filter them out subsequently.

\section{Results and Discussion}

Recall the BPFO calculation from Table 1, the theoretical value of BPFO is 101.6. This number is allowed with $1 \%$ to $2 \%$ change as the rolling elements between bearing races could have some degree of slip during operation. The FFT spectrum of raw vibration signal from test under condition of $1760 \mathrm{~kW}$ minor damaged is presented in Fig. 4. Please be noted that the frequency range is zoomed for BPFO inspection. 


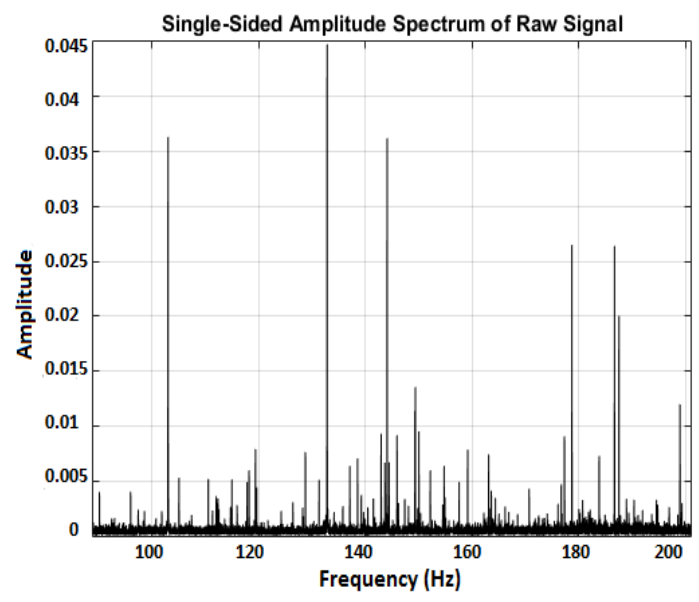

Figure 4. FFT spectrum of raw signal.

The FFT spectrum reveals no fault frequency around $90 \mathrm{~Hz}$. It indicates that the faulty signal is masked by strong background noise and other meshing frequencies. The fault frequency components are not obvious.

Kurtogram is then performed after signal being order tracked. Due to page limitations here only one kurtogram under minor fault condition is illustrated. The kurtogram indicates that maximum impulsiveness has been detected at centre frequency $23800 \mathrm{~Hz}$ with bandwidth of $133 \mathrm{~Hz}$. However optimum centre frequency and bandwidth need some trials and eventually $23650 \mathrm{~Hz}$ and level 6.5 is selected. An optimum band pass filter is then developed based on those parameters. After filtering and zooming, the results of minor and major damage test are presented in Fig. 7 and Fig. 8, respectively. All results indicate that clear defects identifications BPFOs could be observed. The BPFO amplitude of major damage is larger than that of minor damage and also not quite clear. That was because major fault was severe enough to turn into distributed; each roller may have several clicks when rolling over faulty surfaces which cause addictive frequency components around BPFO.

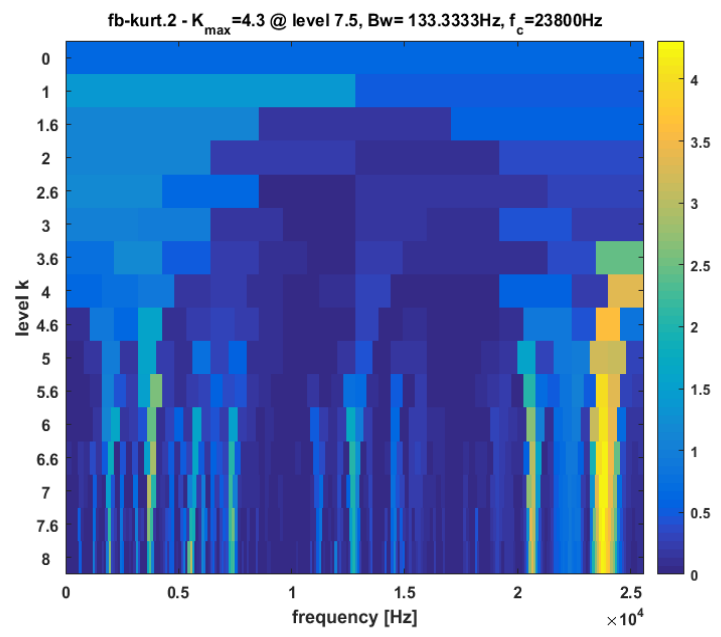

Figure 5. Kurtogram of minor fault condition.
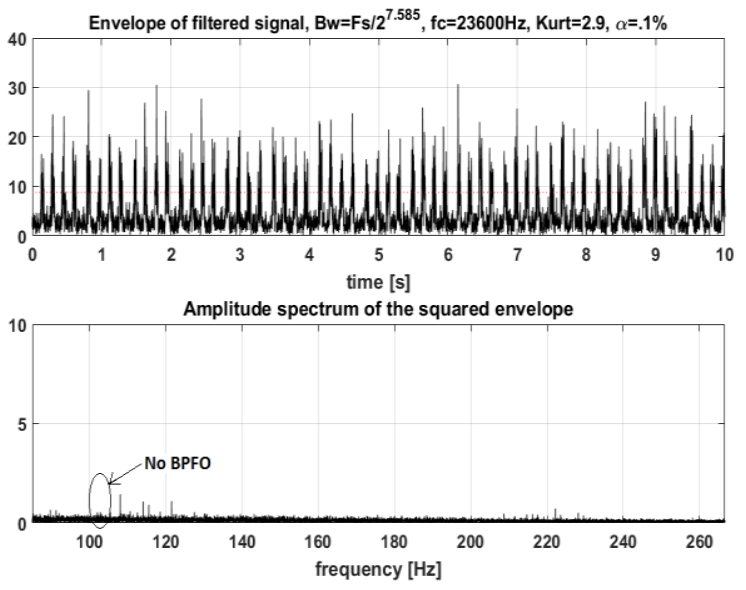

Figure 6. Healthy condition squared envelope spectrum.
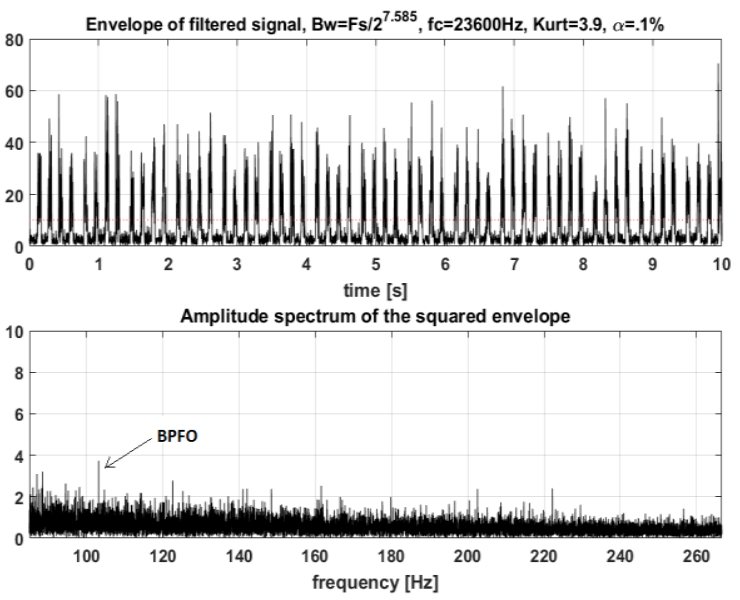

Figure 7. Minor fault condition squared envelope spectrum.

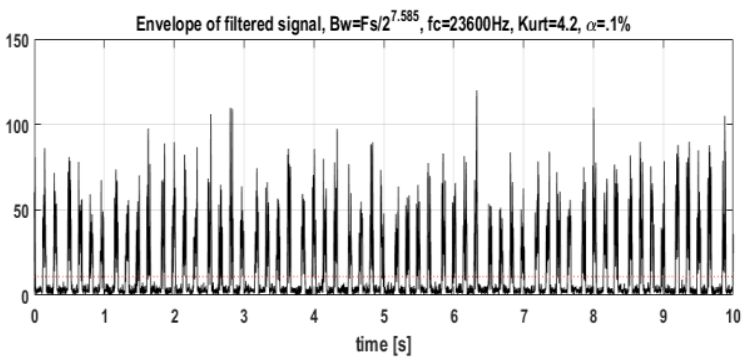

Amplitude spectrum of the squared envelope

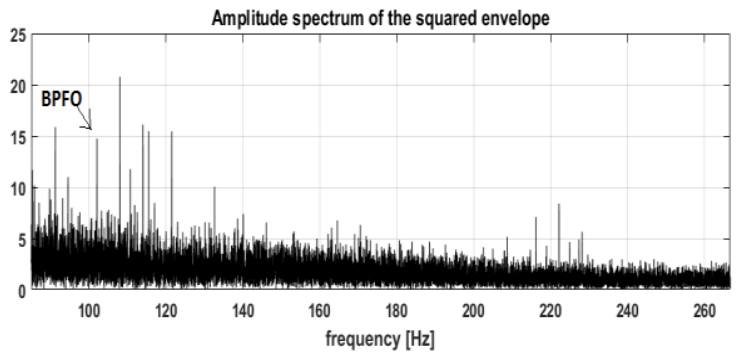

Figure 8. Major fault condition squared snvelope spectrum. 


\section{CONCLUSION}

This paper has described the application of processing technique which combines kurtogram with envelope for analysing experimental data from a commercial helicopter MGB. The proposed technique has gained success in detecting masked frequency components of defect in both minor damaged condition and major damaged condition, which was impracticable with simply applying FFT. This indicates that this technique is capable of countering disturbances resulted from large noises produced in harsh working environments such as a helicopter MGB.

\section{REFERENCES}

[1] M. Lebold, K. McClintic, R. Campbell, C. Byington, and K. Maynard, "Re-view of vibration analysis methods for gearbox diagnostics and prognostics," In Proceedings of the 54th Meeting of the Society for Machinery Failure Prevention Technology (Vol. 634, p. 16), May 2000.

[2] R. B. Randall and J. Antoni, "Rolling element bearing diagnostics-a tutorial," Mechanical Systems and Signal Processing, 25(2), pp.485-520, 2001

[3] J. Antoni and R. B. Randall, "Unsupervised noise cancellation for vibration signals: part I- evaluation of adaptive algorithms," Mechanical Systems and Signal Processing, 18(1), pp.89-101, 2004.

[4] J. Antoni and R. B. Randall, "Unsupervised noise cancellation for vibration signals: part II-a novel frequency-domain algorithm," Mechanical Systems and Signal Processing, 18(1), pp.103-117, 2004.

[5] Department for Transport (2011), "Report on the accident to Aerospatiale (Eurrocopter) AS332 L2 Super Puma, registration G-REDL $11 \mathrm{~nm}$ NE of Peterhead, Scotland, on 1 April 2009," Air Accident Investigation Branch, Aldershot, UK, 2/2011.

[6] M. Greaves, F. Elasha, D. Mba, et. al, "VHM Vibration health or alternative monitoring technologies for helicopters," EASA.2012.OP.13, U.K. Unpublished.
[7] J. Keller and P. Grabill, "Inserted fault vibration monitoring tests for a CH-47D Aft swashplate bearing," In Proceedings of the American helicopter society 61st annual forum, Grapevine, TX, 2005.

[8] M. D. Coats and R. B. Randall, "Order-Tracking with and without a tacho signal for gear fault diagnostics," In Proceedings of Acoustics (pp. 1-6), 2012.

[9] F. Bonnardot, M. El Badaoui, R. B. Randall, J. Daniere, and F. Guillet, "Use of the acceleration signal of a gearbox in order to perform angular resampling (with limited speed fluctuation)," Mechanical Systems and Signal Processing, 19(4), pp.766-785, 2005.

[10] R. Dwyer, "Detection of non-Gaussian signals by frequency domain kurtosis estimation," Acoustics, Speech, and Signal Processing, IEEE International Conference on ICASSP'83. Vol. 8, IEEE, pp. 607, 1983.

[11] F. Elasha, C. Ruiz-Cárcel, D. Mba, G. Kiat, I. Nze, and G. Yebra, "Pitting detec-tion in worm gearboxes with vibration analysis," Engineering Failure Analysis, vol. 42, no. 0, pp. 366-376, 2014.

[12] C. Ruiz-Cárcel, E. Hernani-Ros, Y. Cao, and D. Mba, "Use of spectral kurtosis for improving signal to noise ratio of acoustic ermission signal from defective bearings," Journal of Failure Analysis and Prevention, vol. 14, no. 3, pp. 363-371, 2014.

[13] J. Antoni and R. Randall, "The spectral kurtosis: application to the vibratory sur-veillance and diagnostics of rotating machines," Mechanical Systems and Signal Processing, vol. 20, no. 2, pp. 308-331, 2006.

[14] J. Antoni, "Fast computation of the kurtogram for the detection of transient faults," Mechanical Systems and Signal Processing, vol. 21, no. 1, pp. 108-124, 2007.

[15] F. Bonnardot, R. B. Randall, J. Antoni, and F. Guillet, "Enhanced unsupervised noise cancellation using angular resampling for planetary bearing fault diagnosis," International journal of acoustics and vibration, 9(2), pp.51-60, 2004.

[16] R. B. Randall, "Vibration-based condition monitoring: industrial, aerospace and au-tomotive applications," John Wiley \& Sons, pp. 200202, 2011. 